\title{
Standard primary cytoreduction surgery (CRS) procedure for ovarian cancer (FIGO Stage IIB-IV)
}

\author{
A T Teichmana, Iqra ljaz, Wang Qin, Wang Chunyan, Li Zhu, Chen Yi, Ding Shugui
}

\begin{abstract}
In gynecologic oncology the ovarian cancer has become one of the leading cause of death in women. Overall prognosis for ovarian cancer patients is poor, therefore the management of this condition is restricted to expert multi-disciplinary teams (MDT) in gynecological oncology. Ovarian cancer requires accurate and complete staging so that the metastatic sites are not missed, omitting adequate staging can have significant consequences including a negative impact on survival rates. Thus the goal for survival benefit of ovarian cancer is to surgically remove all visible macroscopic tumor, because the complete cytoreductive surgery (CRS) has been shown to be associated with prolonged survival. In a remarkable number of women, complete CRS is very challenging. Especially in those with many small metastases on the peritoneal and intestinal surfaces. Primary CRS has been in practice for long time
\end{abstract}

as an effective method of treating peritoneal metastases (PM) from ovarian cancer, it comprises extensive peritonectomy procedures and en bloc visceral resection. CRS is a technically challenging surgery that requires a considerable amount of skill and appropriate patient selection. Even though there are several conflicts on primary CRS but due to lack of strong evidence against comparable efficacy of primary CRS with adjuvant chemotherapy and neoadjuvant chemotherapy with interval debulking surgery, the former stays the priority, remaining the preferred method of management and which is practiced in western countries routinely. This article is a review of the CRS techniques we are currently performing for primary CRS, hereby we have described the operative details for removal of cancer in advanced epithelial ovarian cancer patients with widespread pelvic and abdominal involvement.

Key words: ovarian cancer, cytoreductive surgery, peritonectomy, techniques of cytoreductive surgery

Sri Lanka Journal of Obstetrics and Gynaecology 2019; 41: 25-41

DOI: http://doi.org/10.4038/sljog.v41i2.7885

\section{Background}

Ovarian cancer is the third most common gynecological cancer in women worldwide and is the fifth leading cause of cancer-related death ${ }^{1}$. Majority of the females are diagnosed with advanced stage ovarian cancer due to the lack of effective early screening and diagnostic strategies, and at present the chemotherapy and debulking/ cytoreduction surgery (CRS) is the mainstay of therapy. The surgical treatment of ovarian cancer can be categorized

${ }^{a}$ Consultant Obstetrician and Gynaecologist, Sezuan Provincial Center for Gynaecology and Breast-Diseases, Dept. of Perinatal Medicine, Medical University of Southwest China.

Correspondence: ATT, e-mail: <alexander.teichmann@prof-teichmann.de>

Received 24 $4^{\text {th }}$ April 2019 and revised version accepted $2^{\text {nd }}$ June 2019.

Competing interest: The authors report no conflict of interest

This is an open-access article distributed under the terms of the Creative Commons Attribution 4.0 International License, which permits unrestricted use, distribution and reproduction in any medium provided the original author and source are credited. 
into simple and radical/ultra-radical surgical procedure performed as part of primary cytoreduction surgery, main goal of undertaking the CRS is to remove all the visible/ macroscopic tumor, leaving no residual disease behind, which can be achieved by complete/ultra-radical CRS where extensive peritonectomies along with en-bloc resection of the viscera is needed to be performed ${ }^{2,3}$. Another reason to leave no tumor residue behind is related with the effectiveness of upfront chemotherapy, which are nearly ineffective eradicating tumor nodules larger than 2.5 $\mathrm{mm}^{4}$. The progression-free survival (PFS) and overall survival (OS) after complete/ ultra-radical CRS is significantly

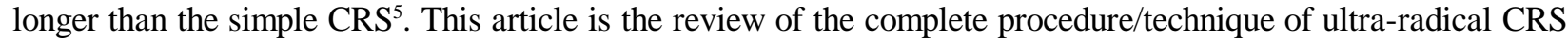
we currently are using in our Gynecologic Oncology Center.

\section{Cytoreduction surgery procedure}

\section{Patient positioning and preparation:}

The patient is placed in the modified lithotomy position with the gluteal fold at the end of the table to allow full access to the perineum and utmost attention is paid to prevent pressure points and myonecrosis of the calf muscles $^{6}$. After general anesthesia, the skin is prepared from the mid-chest to mid-thigh with the preparation of the genitalia. After catheterization, the patient is draped and is now ready to begin the surgery ${ }^{7,8}$.

\section{Exploratory Laparotomy:}

- Abdominal cavity is opened through a vertical extended midline incision from the xiphoid process to the pubic symphysis and incisional site has usually been marked formerly as shown in (Figure 1a), and as always a cautious entry in the abdominal cavity is exercised to prevent injury to intestines, especially at time of nicking and cutting the anterior parietal peritoneum (Figure1b).
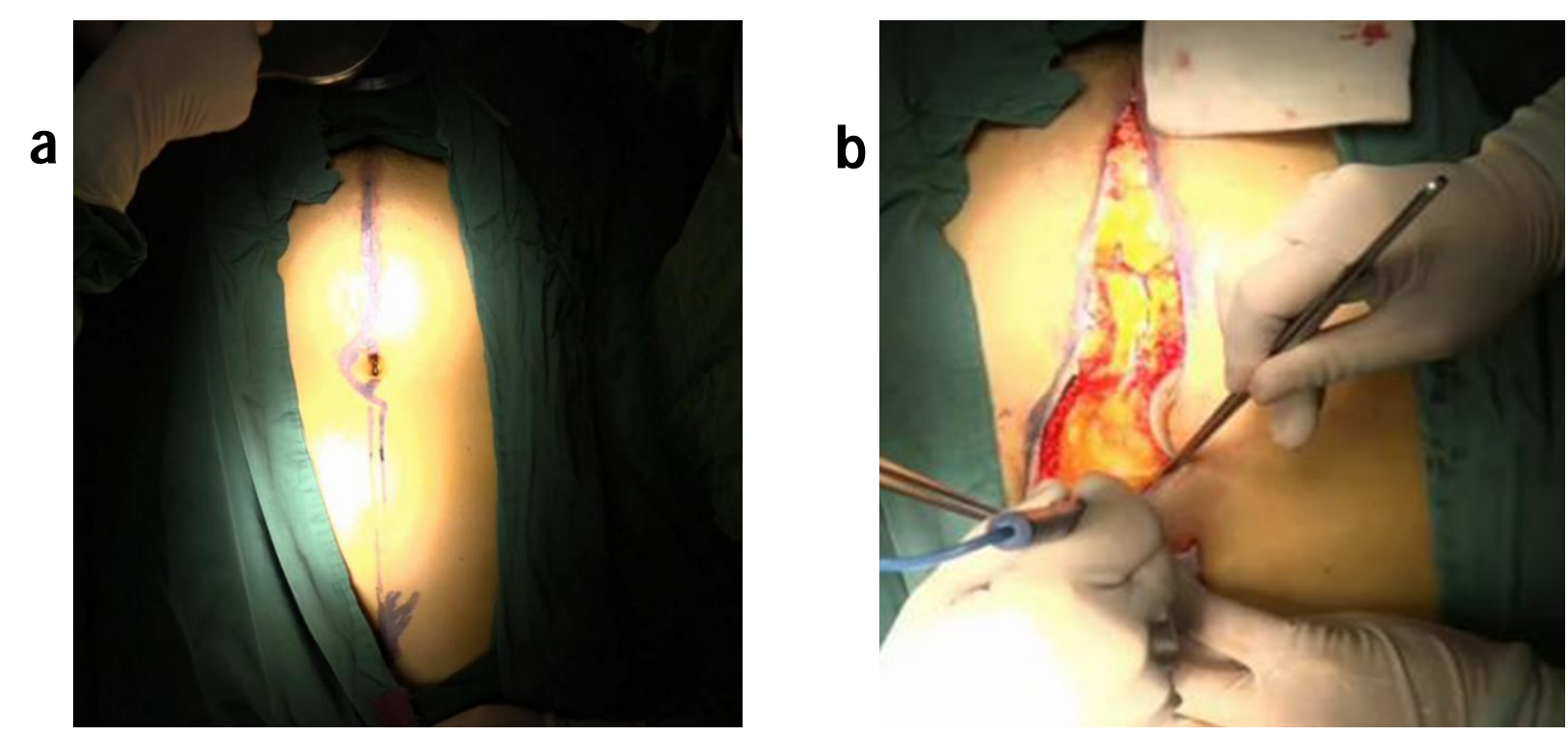

Figure 1. (a) vertical median xipho-pubic incision site marked by drawing a line.

(b) opening up the abdomen.

The abdomen is entered and now for further procedure the adequate abdominal exposure is needed which is achieved by self-retaining retractors Figure 2 a, thus moving on to the next crucial step of total abdominal and pelvic examination (visual inspection and palpation) to explore the tumor lesions on all peritoneal surfaces as well 
as on abdominal and pelvic viscera (Figure $2 b$ ). The probe contents includes: bilateral transverse septum, the surface of the liver, gallbladder, spleen, stomach, duodenum, pancreas, omentum, small intestine from flexor ligament to the ileocecal part of the small intestine and mesenteric root, cecum, colon, paracolic gutters, rectum, para-aortic lymph nodes, pelvic lymph nodes, adnexa, uterus and pelvic peritoneum. All positive results are recorded on surgical documents. As in this case a bulky tumor was seen in the pelvic cavity during pelvic examination (Figure 2c), which was tightly adhered to the anterior abdominal wall (Figure 2d). Suspicious (harboring metastasis) deposits on pelvic and abdominal peritoneum and adhesions are excised/biopsied (in the absence of any suspicious areas random peritoneal biopsies from pelvis, paracolic gutters, undersurfaces of diaphragm and urinary bladder are taken) at this stage and specimens are sent for rapid frozen section for pathological evaluation to assist in further management.
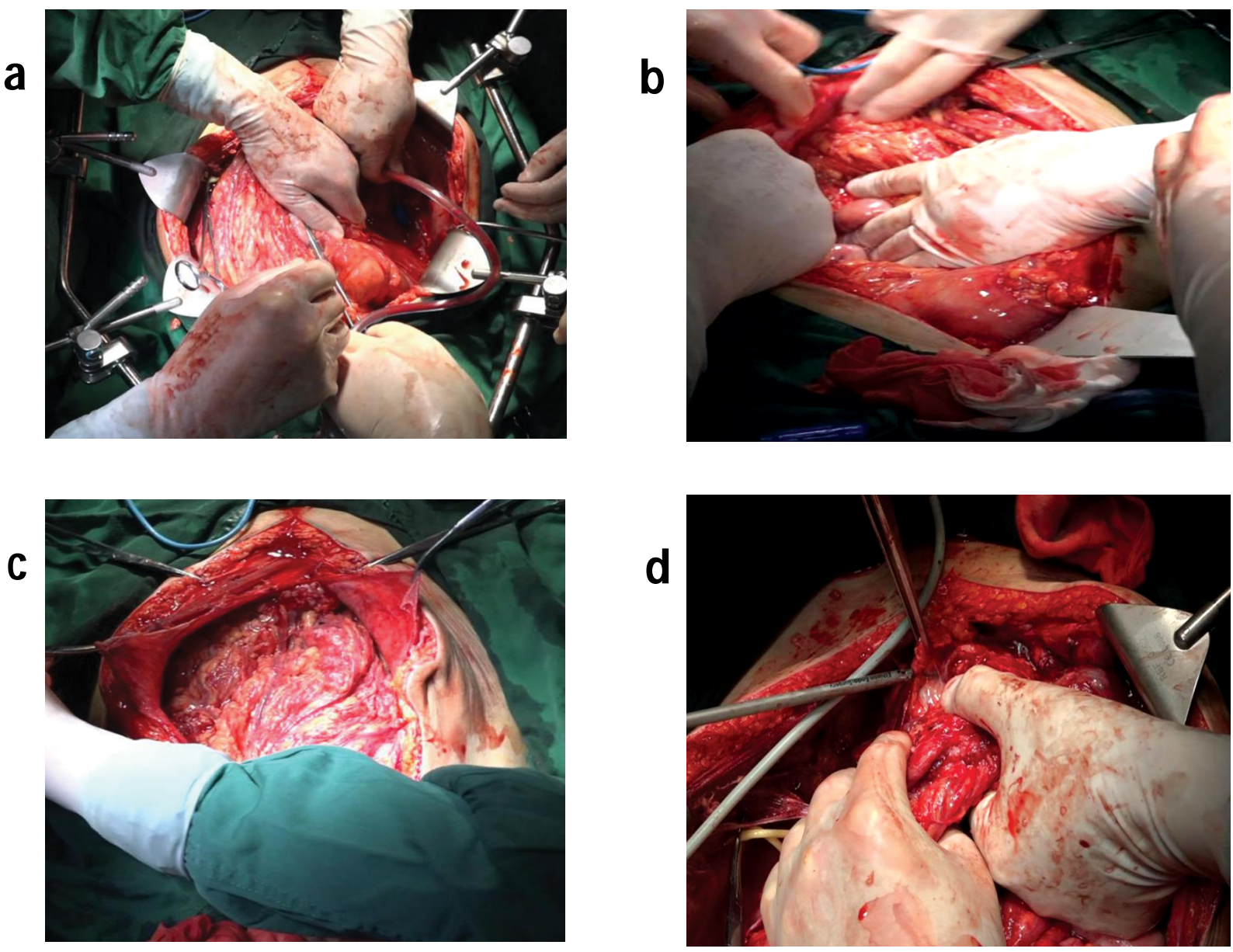

Figure 2. (a) placement of abdominal self-retaining retractor (b) inspection and palpation of abdominopelvic viscera, (c) a bulky tumor is seen in the pelvic cavity, (d) tumor adhesions to the anterior abdominal wall.

\section{En bloc resection of Viscera:}

Thorough exploration enables us for the further step where we perform en bloc resection of viscera, which is an effective procedure of CRS, we begin resection from pelvic organs and it includes resection of extra peritoneal uterus/adnexa/pelvic tumor implants/ pelvic peritoneum (including anterior pelvic peritoneum overlying the bladder and peritoneal reflection of pouch of Douglas POD)/abdominal peritoneum (including anterolateral parietal peritoneum, paracolic gutters and right/left subphrenic peritoneum) (if the rectum is involved, then it is separated and resected) $)^{9,10}$. 
- We begin with pelvic peritonectomy: for which at first the peritoneum of the lower anterior abdominal wall is stripped off the underlying muscles from the midline on either side (Figure $3 a, b$ ) to fully expose the extraperitoneal space, revealing the ureters / ovarian blood vessels / iliac blood vessel / uterine vessels and round ligament.
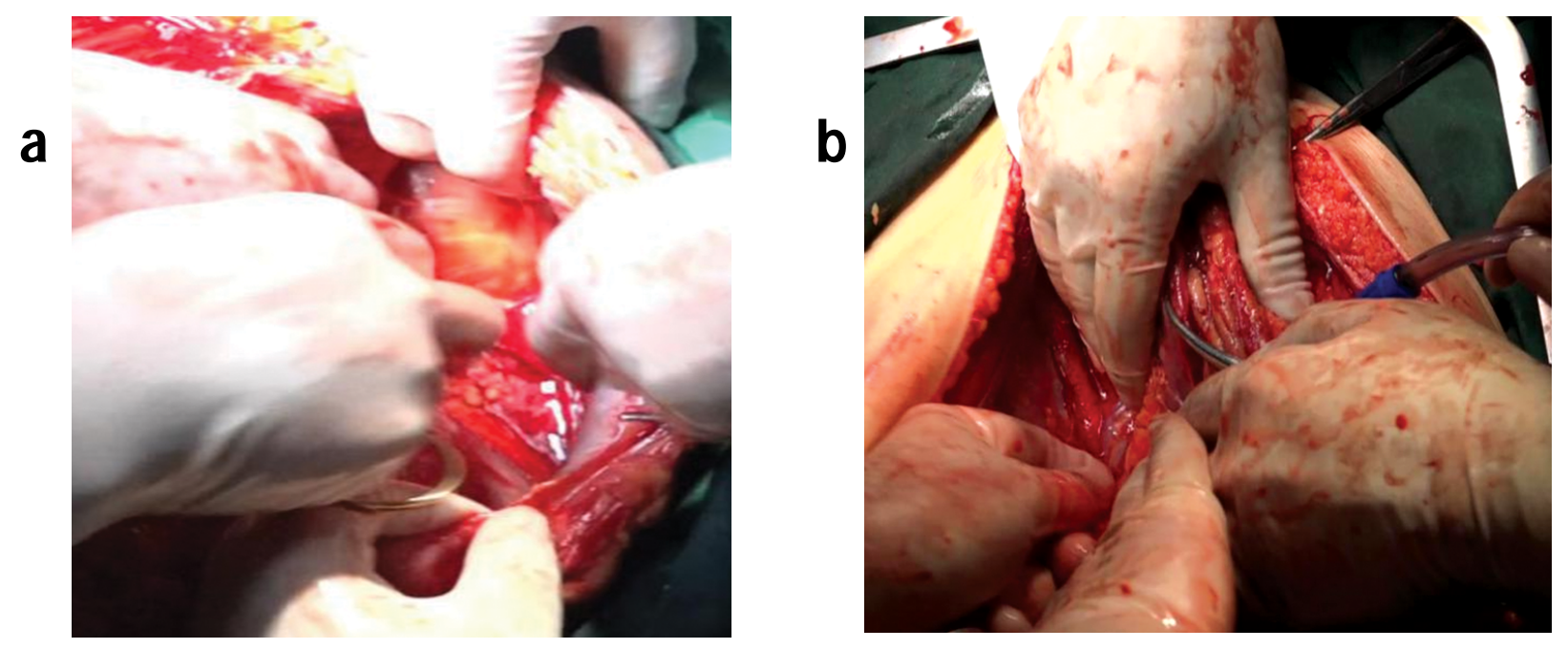

Figure 3. Stripping off the pelvic peritoneum to expose extraperitoneal space (a) right side (b) left side.

The following steps are performed in the extraperitoneal cavity: for ureterolysis, ureters are identified and dissected away from the peritoneum and are marked with silicon tube sling (Figure 4 a, b) to avoid confusion with other vascular, ligamentous structure and adhesions as well.
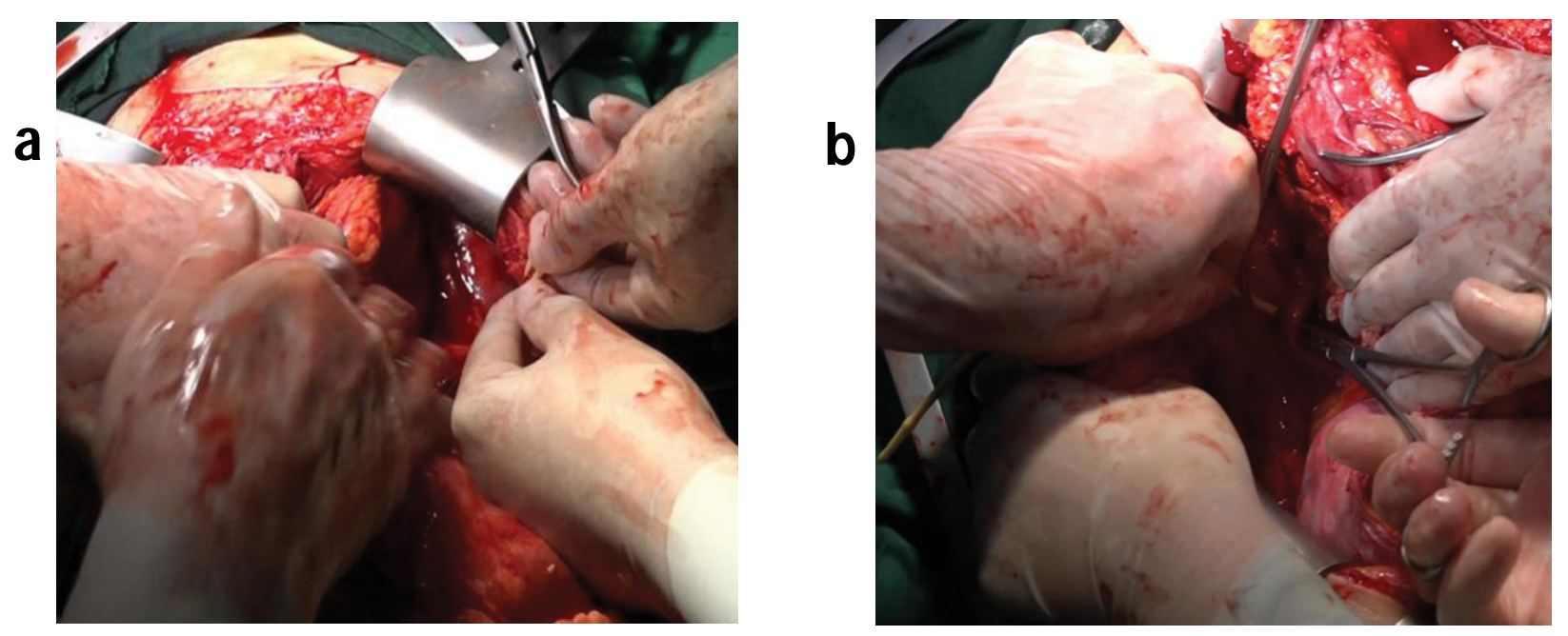

Figure 4. (a) right ureterolysis and marked with a silicon catheter (b) left ureterolysis.

Once the ureters have been now the high ligature and severing the ovarian vessels is performed (Figure $5 \mathrm{a}, \mathrm{b}$ ), (the left ovarian vein is ligated near the left renal vein and on the right side it is ligated near the inferior vena cava, the ovarian arteries are ligated and cut off near the abdominal aorta). 

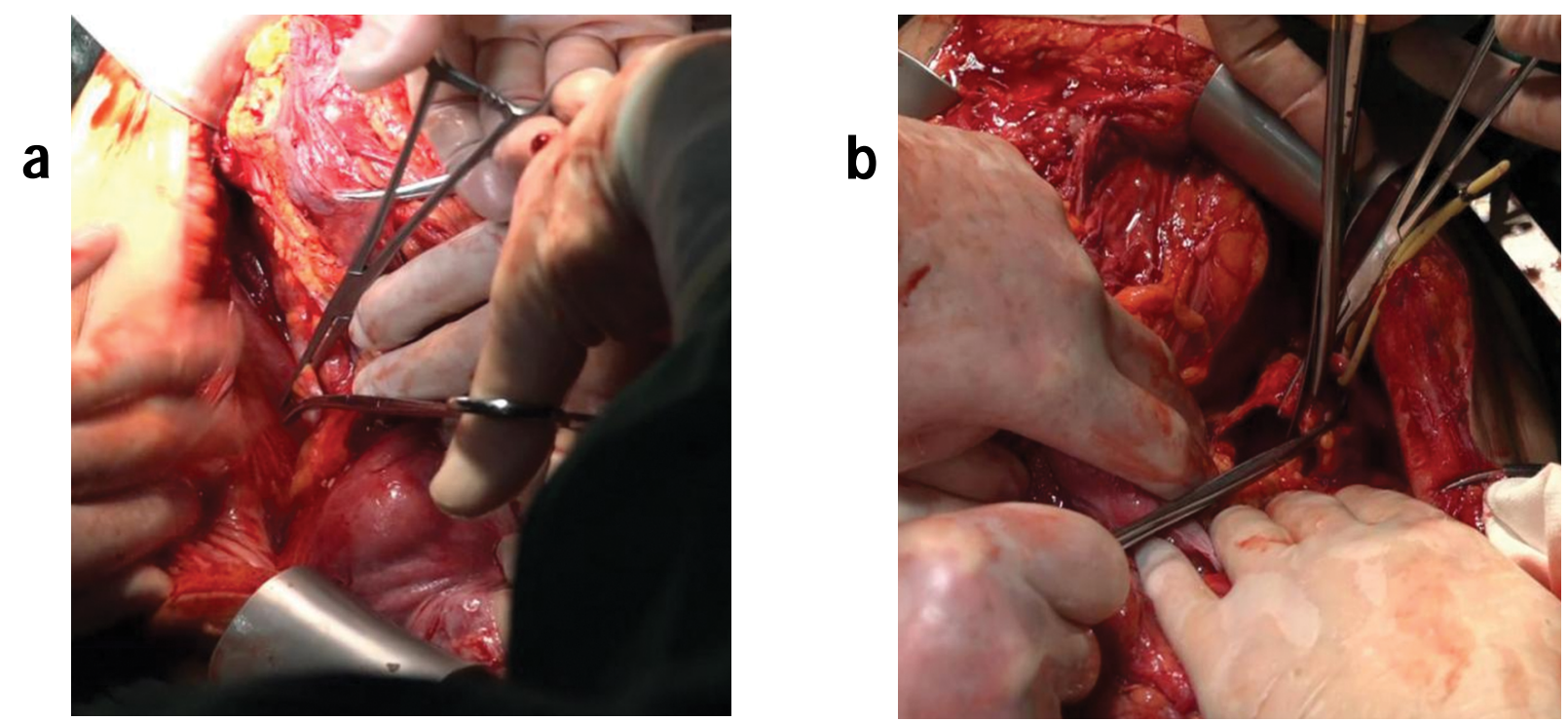

Figure 5. (a) separate (mobilize), clamp, cut, ligature of left ovarian blood vessels at the high site (b) right ovarian.

The infundibulopelvic ligaments are divided on either side at just proximal to the pelvic wall. (Figure 6a) shows the treatment of left uterine round ligament.

The uterine vessels are exposed by deeper dissection extraperitoneally and are then clamped severed and ligated using silk suture on either side just medial to the ureters (Figure 6b).
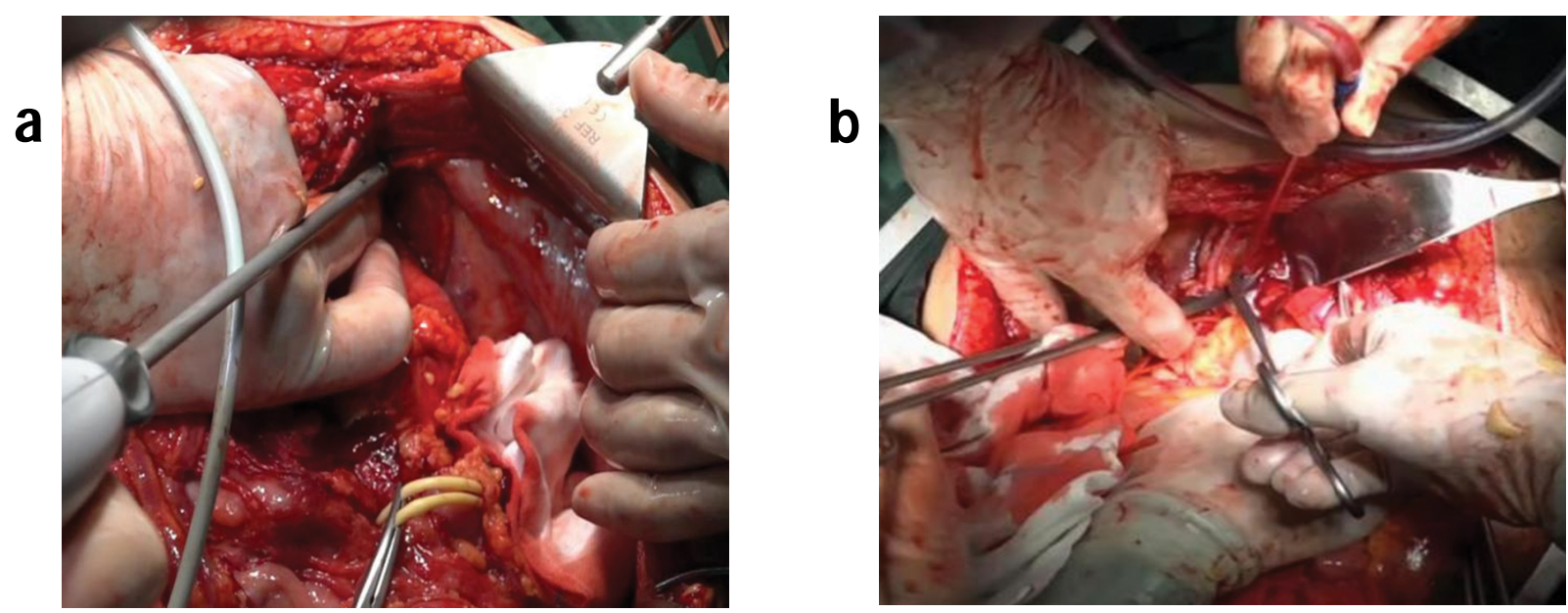

Figure 6. (a) left infundibulopelvic ligament divided proximal to the pelvic wall, right side (figure omitted), (b) left uterine vessels divided just medial to the ureter, right uterine vessels (figure omitted).

For the bladder peritonectomy initially we dissect parietal peritoneum overlying on top of it and instill $200 \mathrm{ml}$ normal saline with methylene blue in the bladder and now further bladder parietal peritoneum is stripped to the extent required (partial cystectomy is feasible if the muscularis is invaded by tumor and bladder repair surgery is performed afterward) and then the bladder is mobilized caudally to develop vesicovaginal space (Figure $7 \mathrm{a}, \mathrm{b}$ ). 

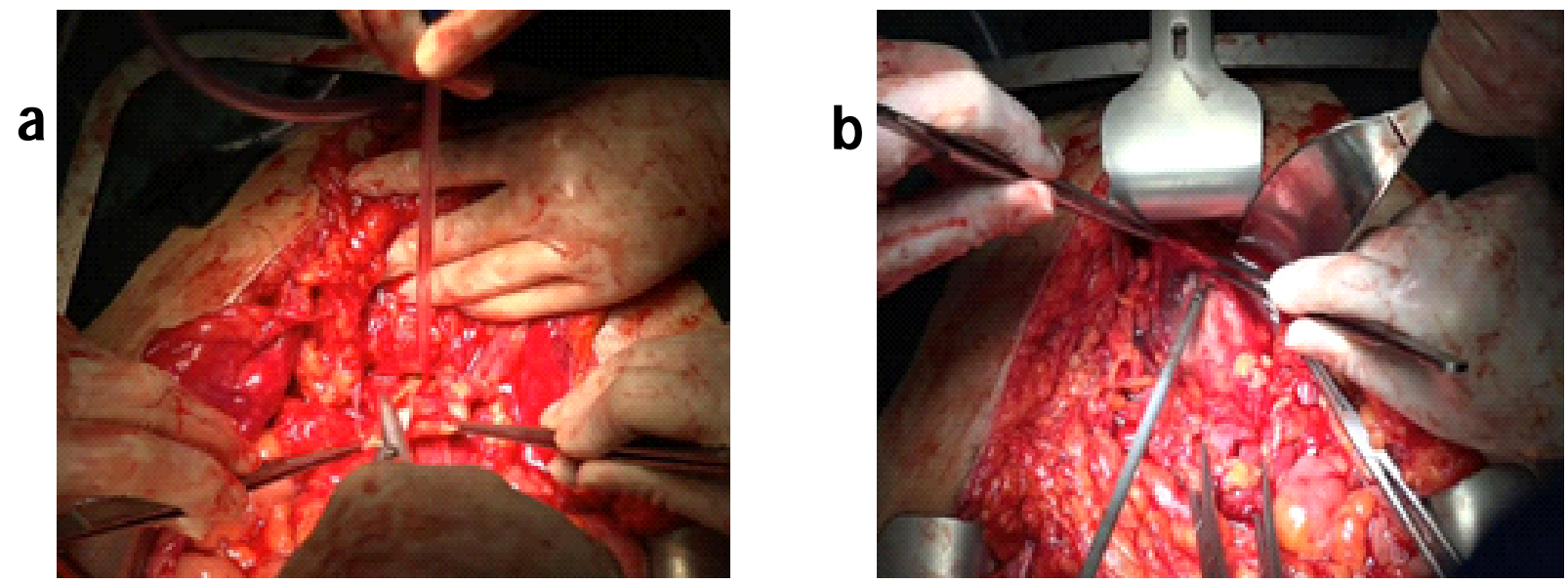

Figure 7. (a) prevesical peritonectomy (b) Mobilizing (pushing down) bladder from the cervix to external cervical orifice to create vesicovaginal space.

Once enough space has been achieved between bladder and vagina, the anterior vaginal wall is dissected (Figure $8 \mathrm{a}$ ) and vaginal cuff is divided circumferentially along the vaginal fornix ring (Figure $8 \mathrm{~b}$ ), the rectovaginal septum is exposed. The perirectal fat is divided beneath the peritoneal reflection of the pouch of Douglas.
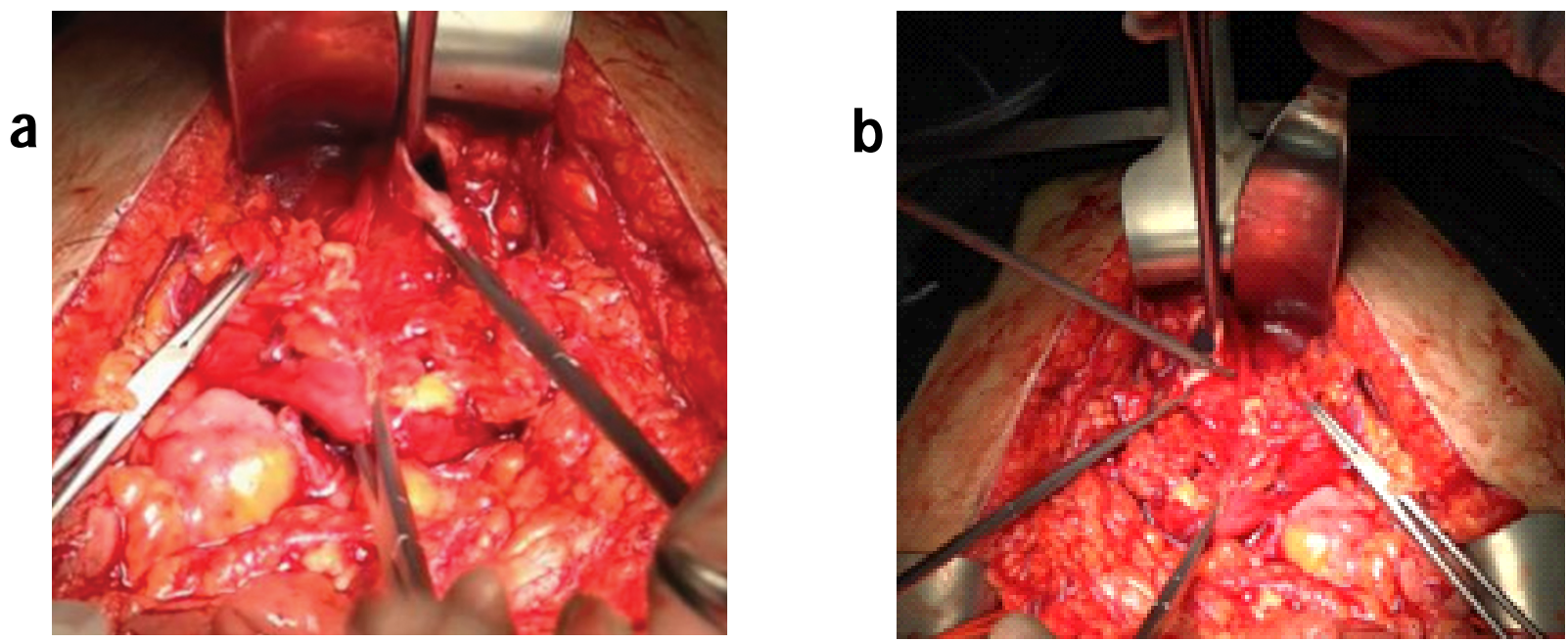

Figure 8. (a) incision of the anterior vaginal wall, (b) vaginal circumcision.

Moving along the ureteral pathway the uterosacral ligament complex is clamped, severed and sutured (Figure $9 \mathrm{a}, \mathrm{b}$ ). 

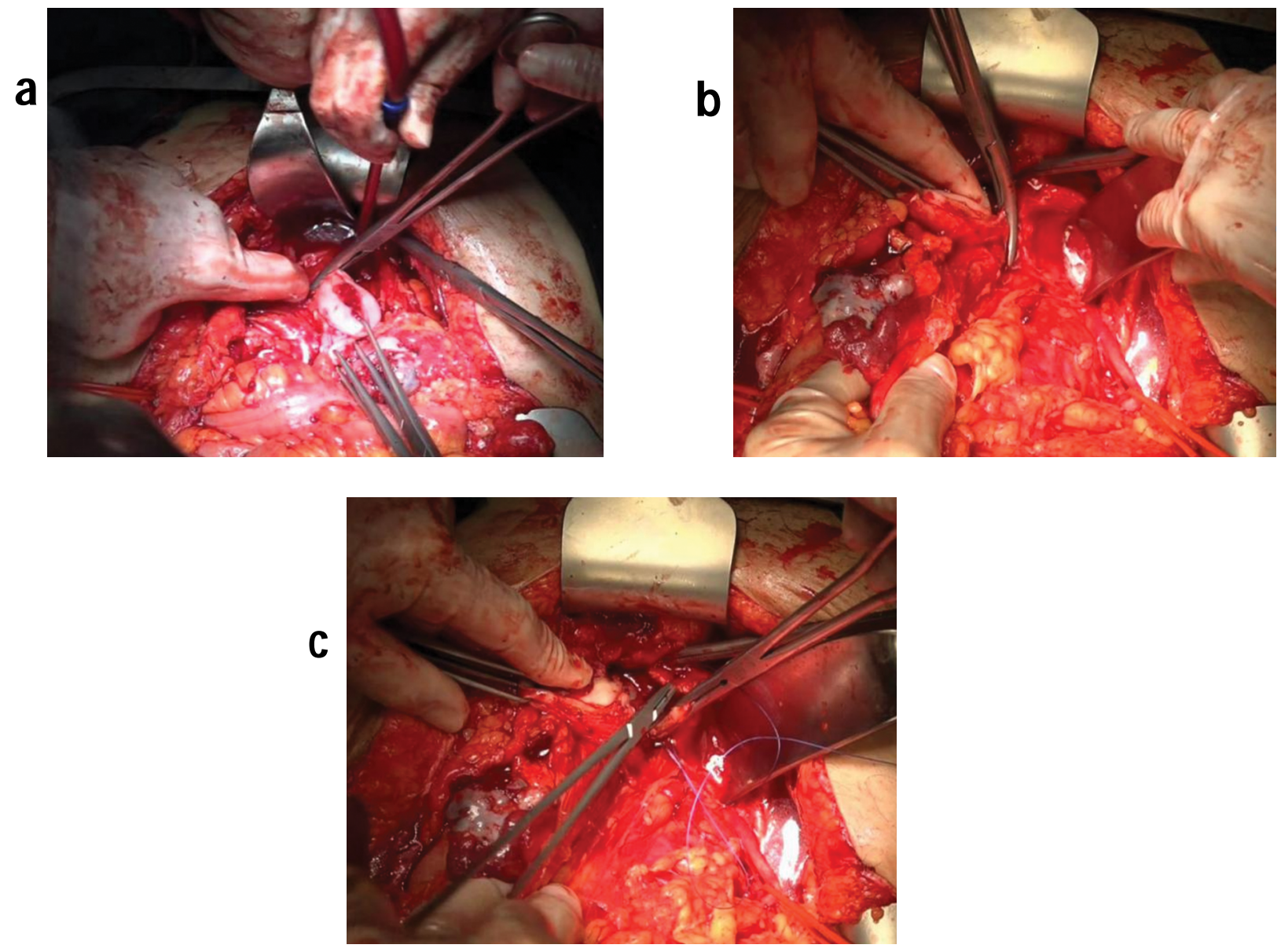

Figure 9. Clamp, cut and suture the uterosacral ligament complex (a) left side (b) right side, (c) suturing the right uterosacral ligament complex.

The vaginal stump is then closed at this stage (Figure 10).

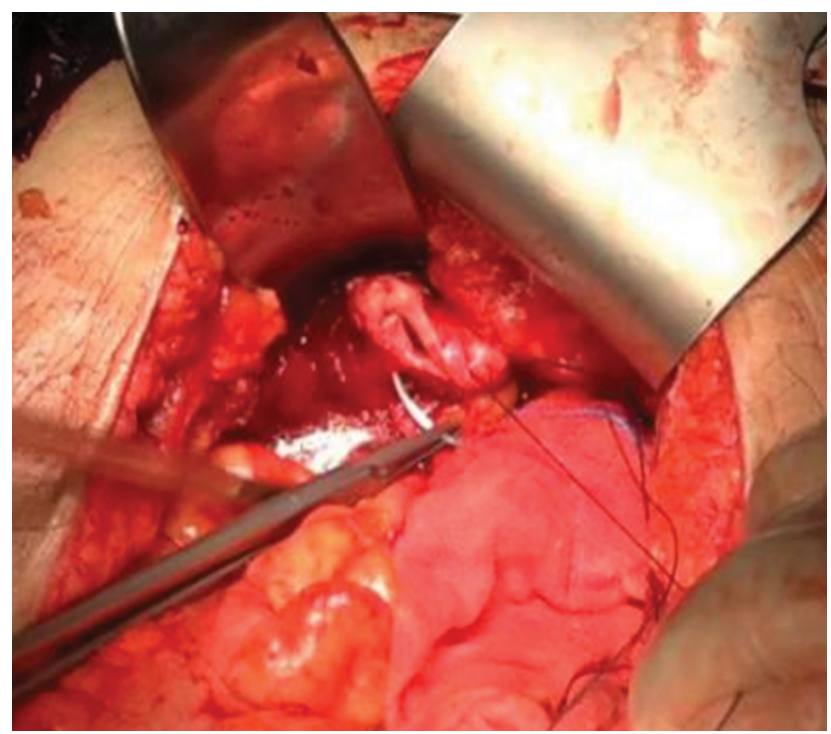

Figure 10. Suture closure of vaginal stump. 
Now the peritoneum is stripped on both sides of the rectum to create the pararectal and behind, the retrorectal space which is further dissected down to the level of the pelvic floor (Figure $11 \mathrm{a}, \mathrm{b}$ ). The posterior dissection is progressed and moves to the right and the left sides of the rectum and is mobilized (Figure $11 \mathrm{c}$ ). To separate the colon the paracolic gutter peritoneum is stripped away. If cancer has invaded the rectum and the sigmoid colon, it is resected at this stage (Figure $11 \mathrm{~d}$ ), and then the upper and lower ends of bowel are anastomosed ${ }^{11}$. Hysterectomy is completed in a retrograde fashion, pelvic peritonectomy along en bloc resection of pelvic viscera is completed (Figure 12) and we can remove the surgical specimen now or afterward (where pelvic peritonectomy is continuous with lateral abdominal wall peritonectomy and so on) as we have shown later in the article.

a

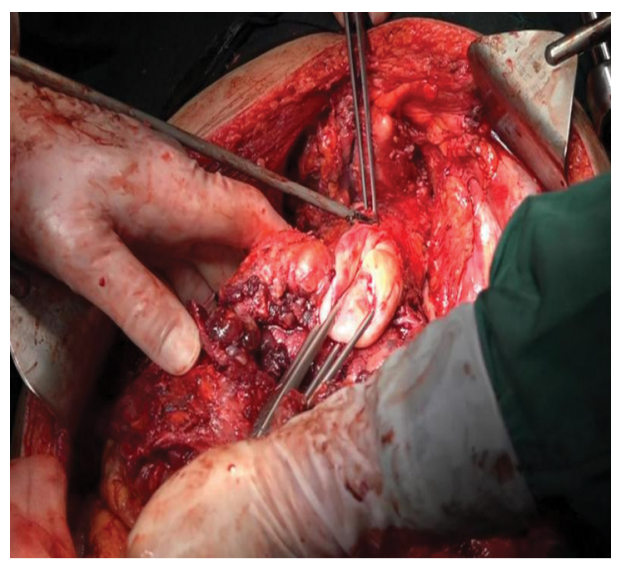

C

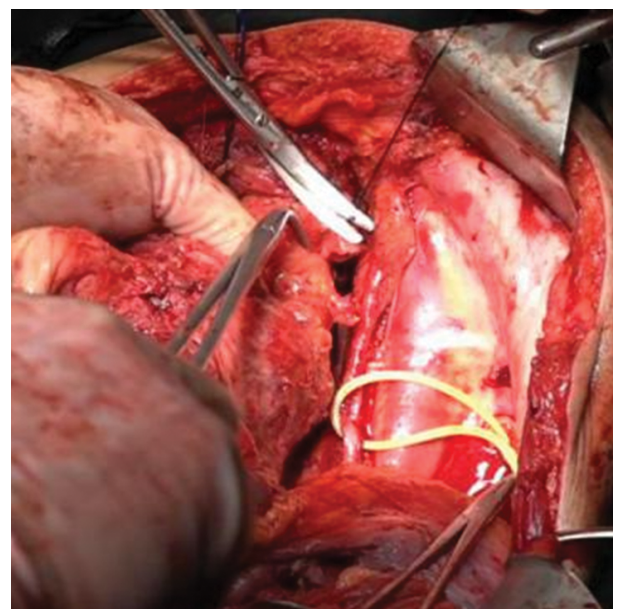

b
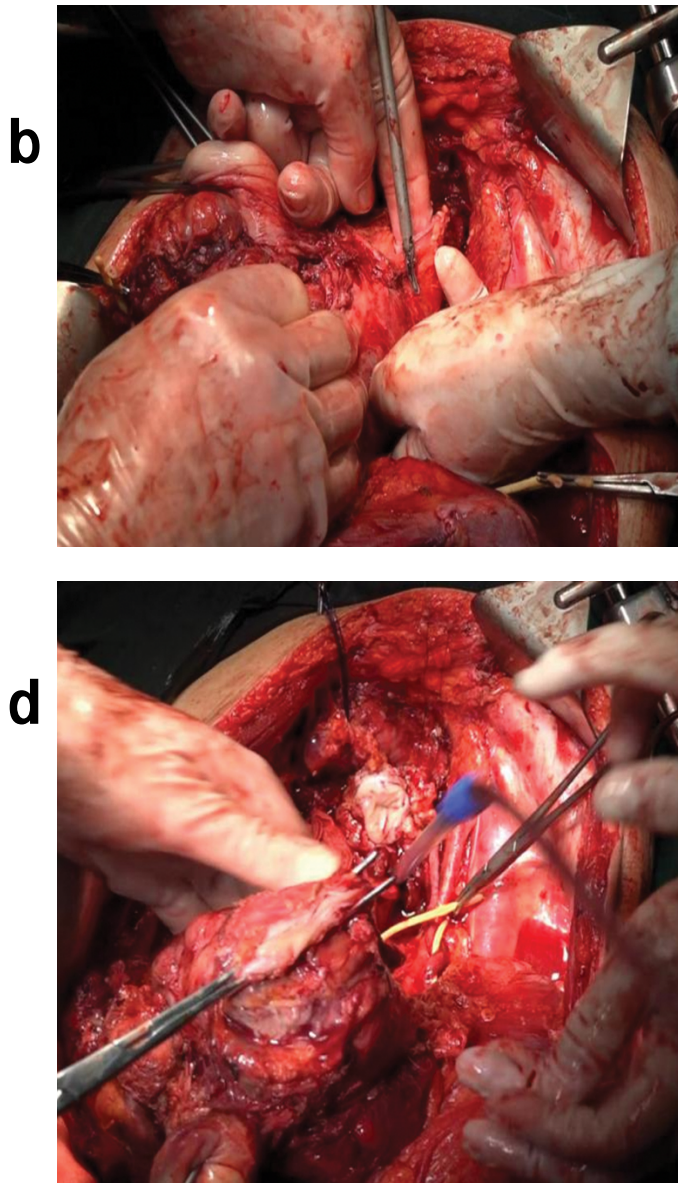

Figure 11. (a) creating rectovaginal space, (b) separate perirectal tissue (c) mobilizing the rectum (d) resection of the rectum under the tumor involvement.

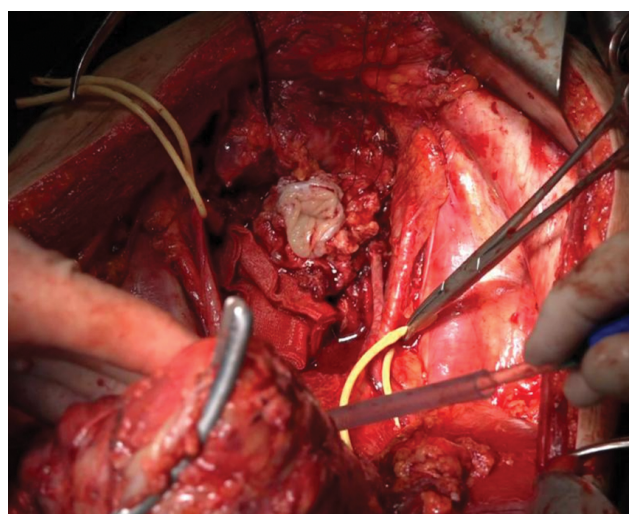

Figure 12. En bloc uterus, adnexa, rectum, and pelvic peritoneum, and parietal peritoneum on top of the bladder removing from the pelvis. 
After resection of pelvic organs the next major step is to deal with bowel metastases, as the mesentery of the small intestine is a frequent site of peritoneal metastases and this is amenable to treatment by electrovaporisation and careful dissection to avoid injury to the underlying vessels. If it is possible to achieve cytoreduction to R0 through small and large intestine resection, then we perform the resection of involving intestine. For segmental bowel resection harboring tumor, at first the mesenteric vessels are ligated and divided (Figure 13a) and then the margin of bowel and mesentery on either side is resected (Figure $13 \mathrm{~b}$ ), and the ends of bowel is anastomosed (Figure $13 \mathrm{c}$ ).
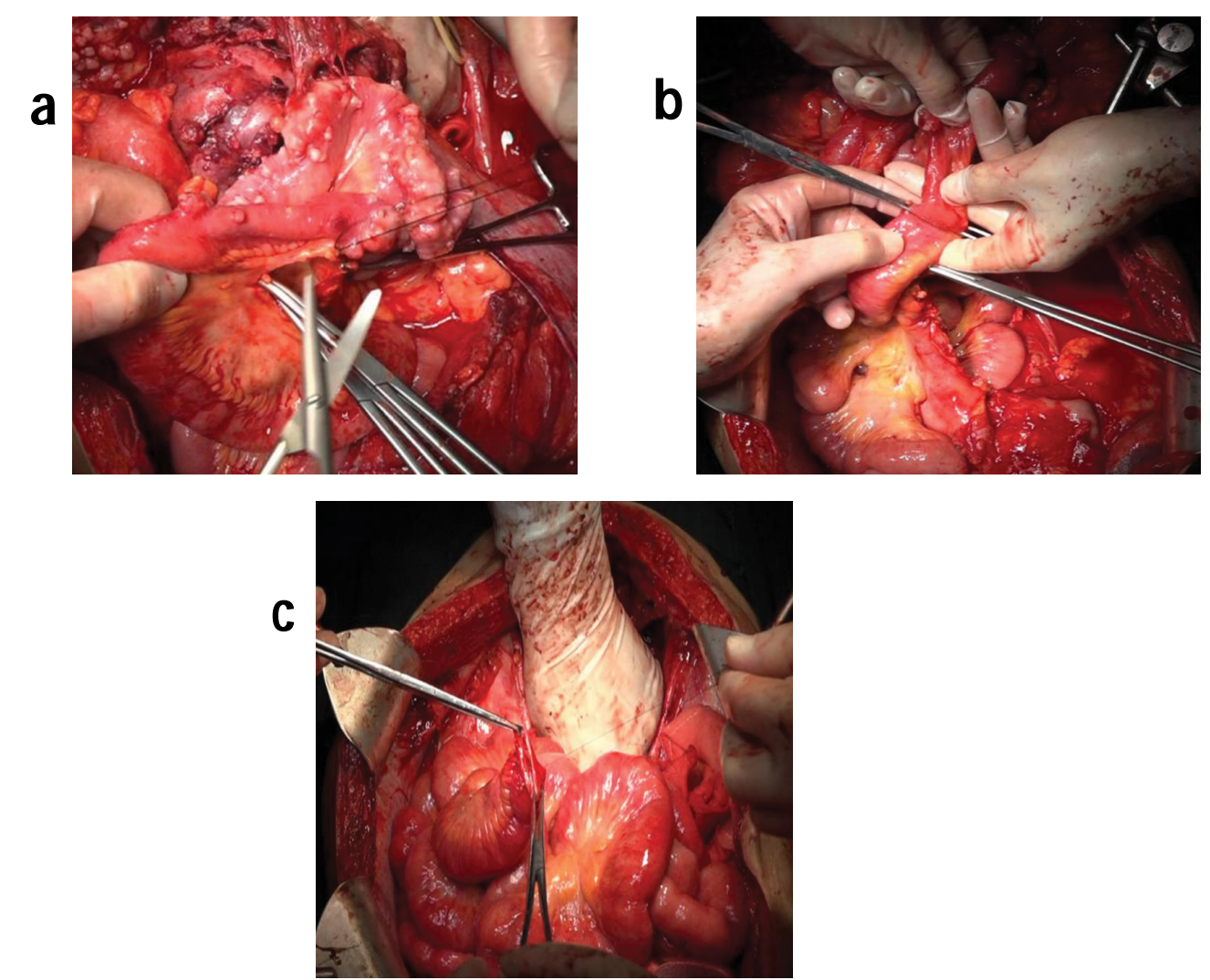

Figure 13. (a) separation, clamping, severing and ligation of the mesenteric vessels (b) resection of the small intestine segment invaded by the tumor, (c) preparation of intestinal anastomosis.

Resection of the ascending or transverse colon is often required in patients with extensive omental deposits or deep bowel and mesenteric deposits. In cases of more extensive disease, a total or subtotal colectomy is required and when 10-15 cm of the rectum is preserved, an ileostomy can better be avoided and the ileorectal anastomosis is performed using the technique of inverting the stapled anastomosis with a layer of interrupted silk sutures 12-14 (Figure $14 \mathrm{a}, \mathrm{b})$. While performing stapled anastomosis care is taken to avoid the left ureter and the vaginal stump injury.
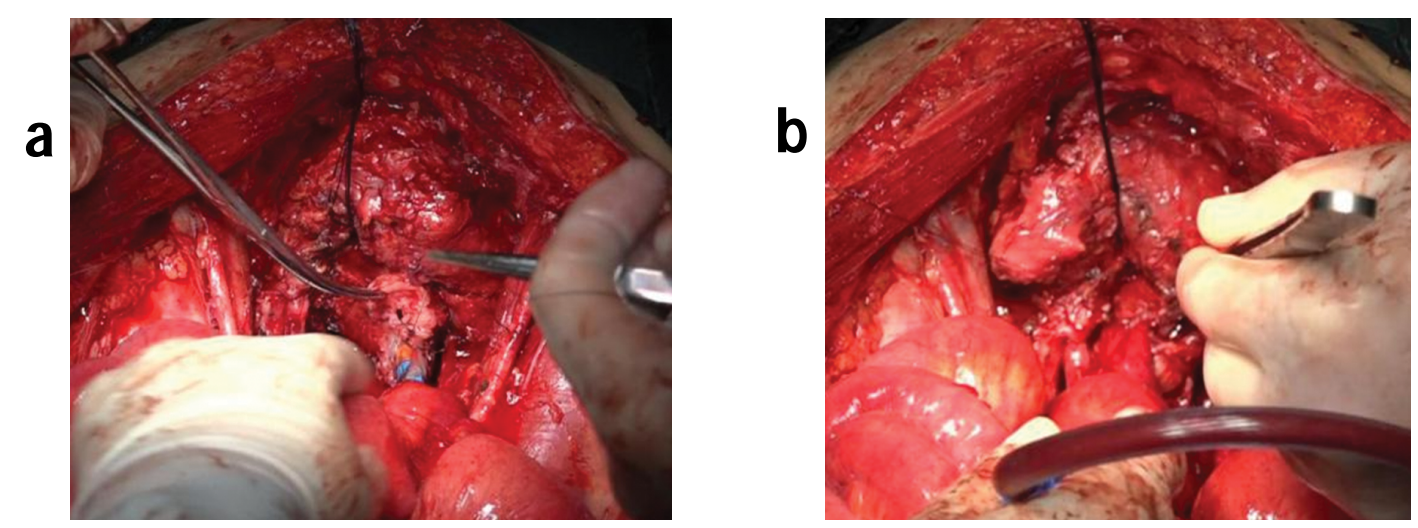

Figure 14. Ileorectal anastomosis (a) before and (b) after. 
Now to resect the upper abdominal tissue and subphrenic diaphragm invaded with tumor, we begin with division of the falciform ligament and triangular ligament of the liver to fully expose superior diaphragmatic area/ bare area of the liver, where the diaphragmatic peritoneum turns onto and becomes continuous with the Glisson's capsule (Figure 15 a). In cases where we encounter heavy disease over the liver, which can form a thick layer over the Glisson's capsule, we remove Glisson's capsule or liver parenchyma depending on tumor invasion using either sharp or electrocautery, the sub-Glissonian space is entered and then by bluntly moving the fingers in this plane, the Glisson's capsule along with the disease can be effectively lifted off the liver surface ${ }^{15-17}$. Isolated deposits of tumors in this area are electrovaporated and dissected off (Figure 15b). Tumor deposits over the caudate lobe and round ligaments are dissected off and electrovaporated in cases showing heavy metastasis to these regions (Figure $15 \mathrm{c}, \mathrm{d}, \mathrm{e}$ ). Cholecystectomy is performed when necessary.
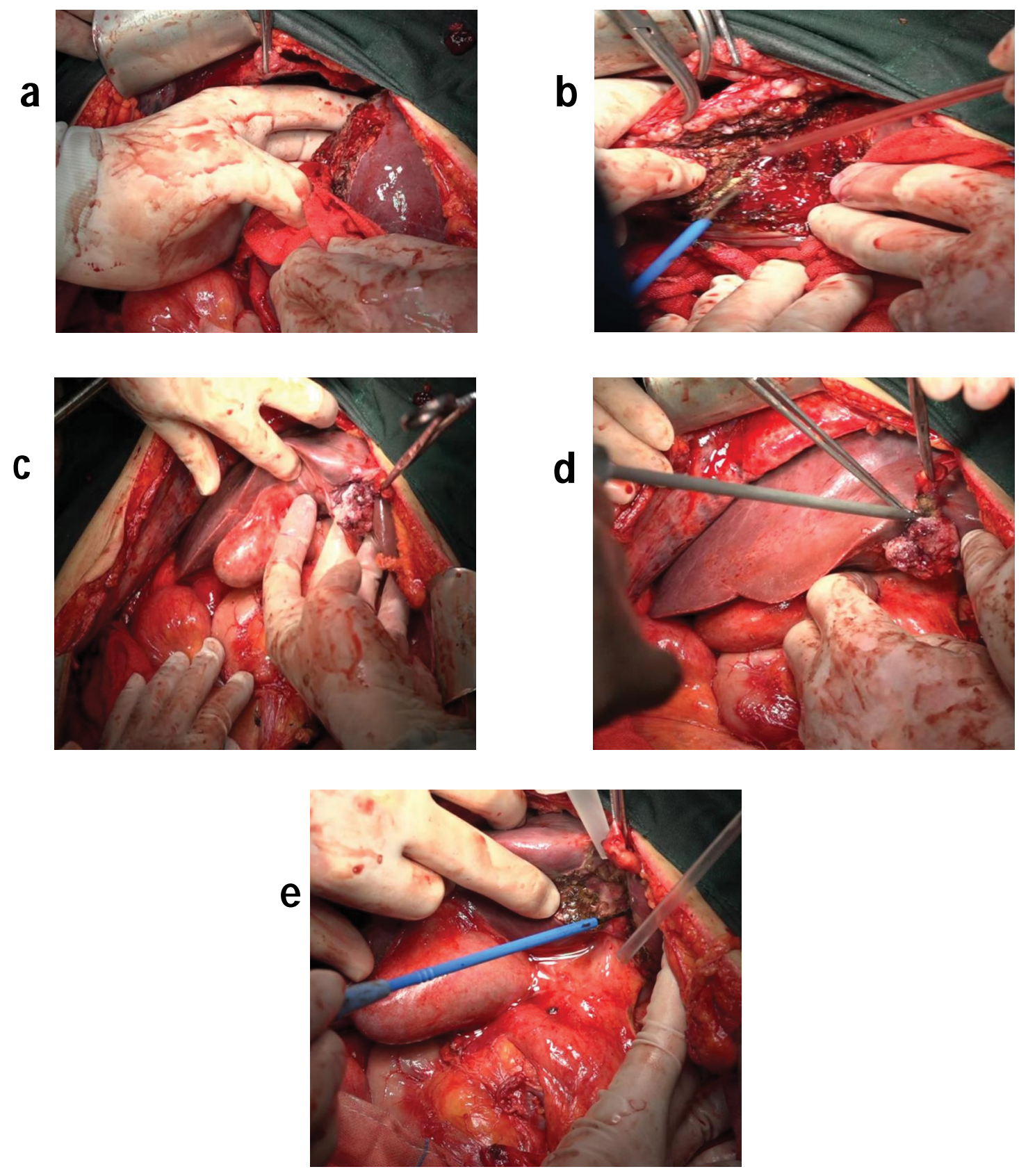

Figure 15. (a) resection of the right triangular ligament, (b) excision of the peritoneum at the junction of the diaphragm and right liver and stripping of the Glisson's capsule,

(c) liver caudate lobe lesion dissection, (d) excision of lesions at round ligament of liver, (e) after excision of lesions at round ligament of liver. 
The diaphragmatic peritoneum is stripped off on the right subphrenic area, which can follow the previous paracolic sulcus peritonectomy continuation (bilateral anterolateral parietal peritonectomy can be performed by the extraperitoneal approach $)^{18}$ (Figure $16 \mathrm{a}, \mathrm{b}$ ). In one case at this point when complete peritonectomy is done the surgical specimen is removed en bloc comprising uterus, adnexa, partial rectum, total colon, partial ileum, omentum, pelvic peritoneum, prevesical peritoneum, abdominal wall peritoneum, bilateral paracolic gutter peritoneum, right subphrenic peritoneum as shown in (Figure 16c).
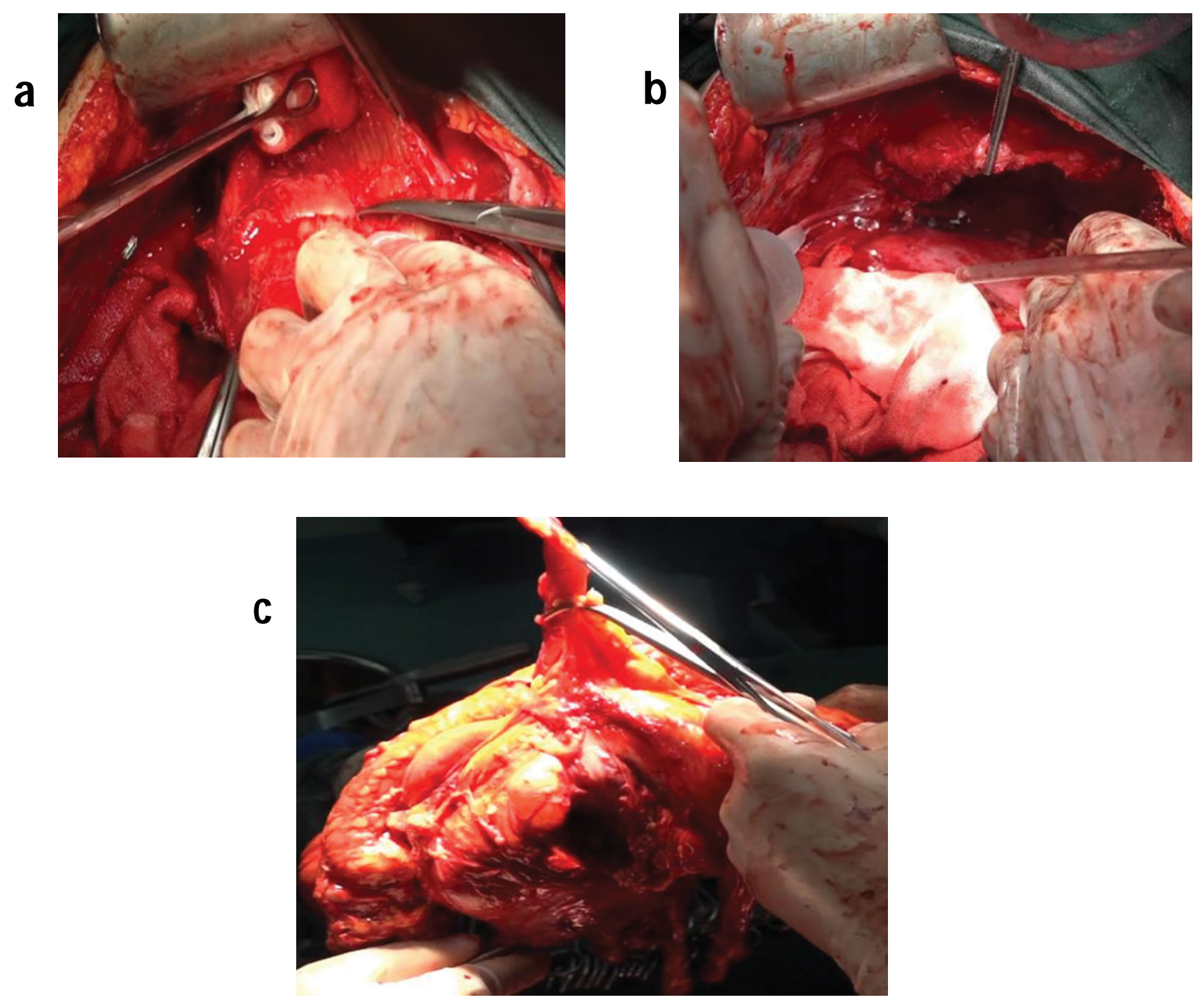

Figure 16. (a) stripping off the right subphrenic peritoneum (b) after right subphrenic peritonectomy and resection of liver lesions (c) surgical specimens en bloc with uterus, adnexa, partial rectum, total colon, partial ileum, omentum, pelvic peritoneum, prevesical peritoneum, abdominal wall peritoneum, bilateral paracolic gutter peritoneum, right subphrenic peritoneum.

Where the tumor deposits are seen to involve the diaphragmatic muscle deeply, it requires the full thickness resection of a part of the diaphragm as this case, shown in (Figure 17a) after the resection of involved diaphragm a thoracic drainage is placed (Figure 17b) and closed the defect with continuous non-absorbable monofilament suture $^{18}$ (Figure $17 \mathrm{c}, \mathrm{d}$ ). 

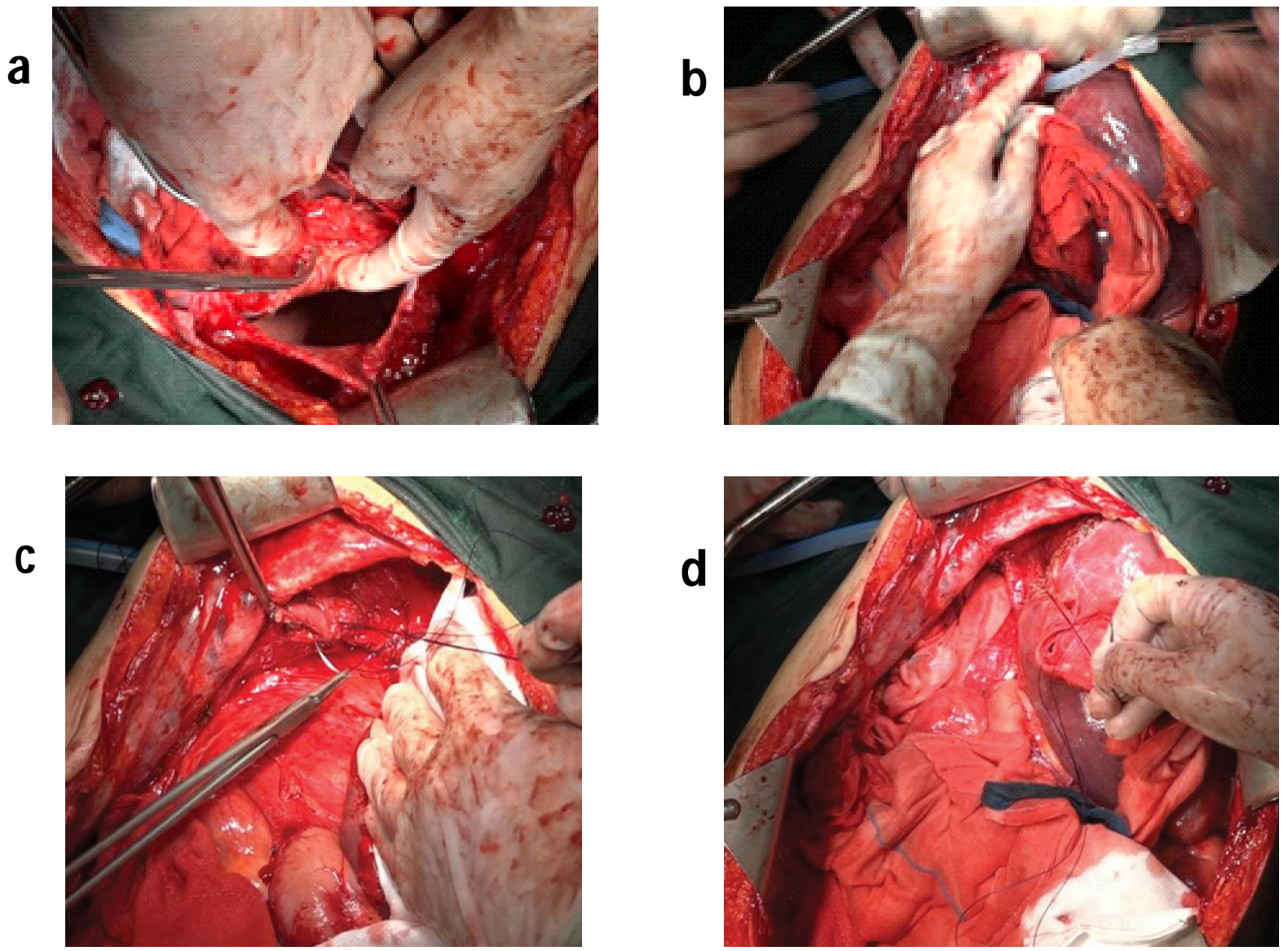

Figure 17. (a) resection of tumor invading deep into the diaphragm, (b) placing thoracic drainage, (c) before suturing the diaphragm, (d) after suturing the diaphragm.

Subgastric omentectomy is performed by dissecting the gastro colic ligament below the stomach also the gastrosplenic ligament is excised on the left (Figure 18a) (care is taken to avoid splenic laceration and bleeding while dealing with splenic ligament), as well as the omental bursa is exposed, examined the lesser omentum and the retroperitoneal organs of the upper abdomen ${ }^{19,20}$. In some cases, the resection of the lesser/greater omentum is performed to achieve tumor-free area (Figure 18b).
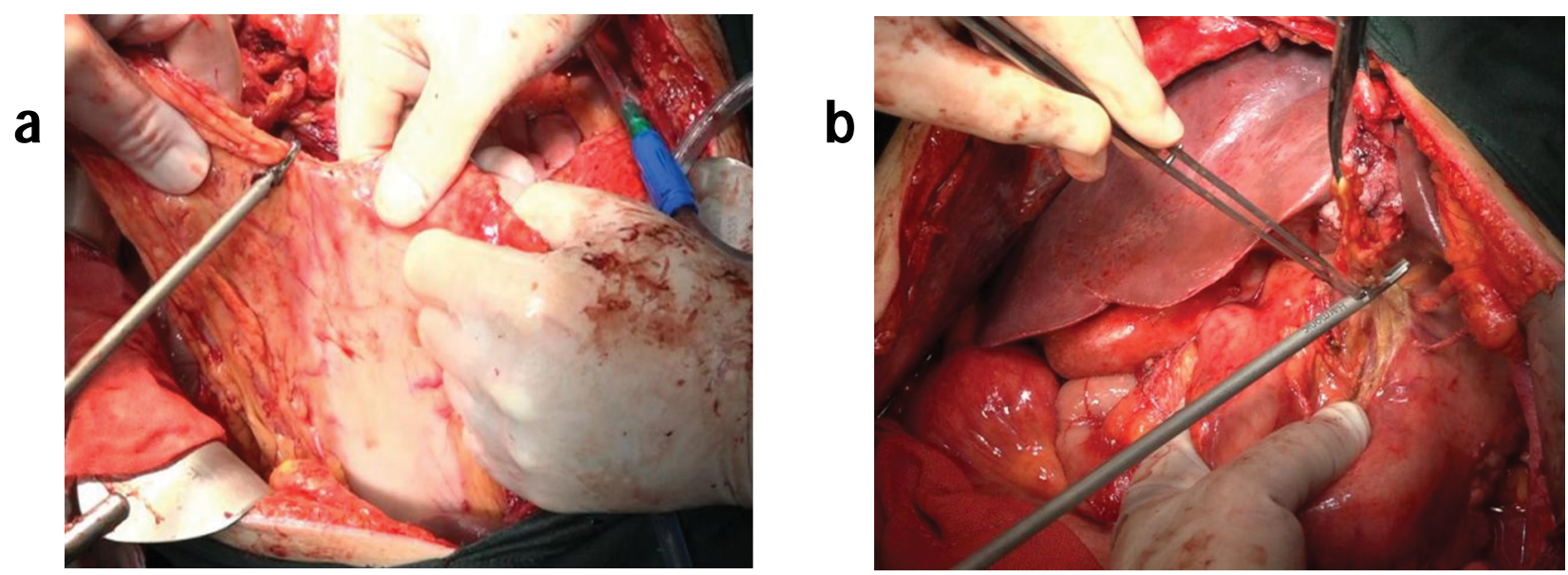

Figure 18. (a) subgastric omentectomy (b) resection of tumor in lesser omentum. 
Appendectomy (Figure $19 \mathrm{a}, \mathrm{b}$ ) is carried out in cases with abnormal or non-serous carcinoma. The cecum and the appendix are found down the right colon strip, the mesoappendix is clamped, divided, ligated and the cecum muscularis is sutured $0.6 \mathrm{~cm}$ away from the base of the appendix using silk suture ${ }^{21}$. The base of the appendix is clamped $0.4 \mathrm{~cm}$ away from the ligation line, cut off and ligated. The appendix stumps is disinfected with alcohol and saline, tighten the suture line, and embedded the appendix stump.
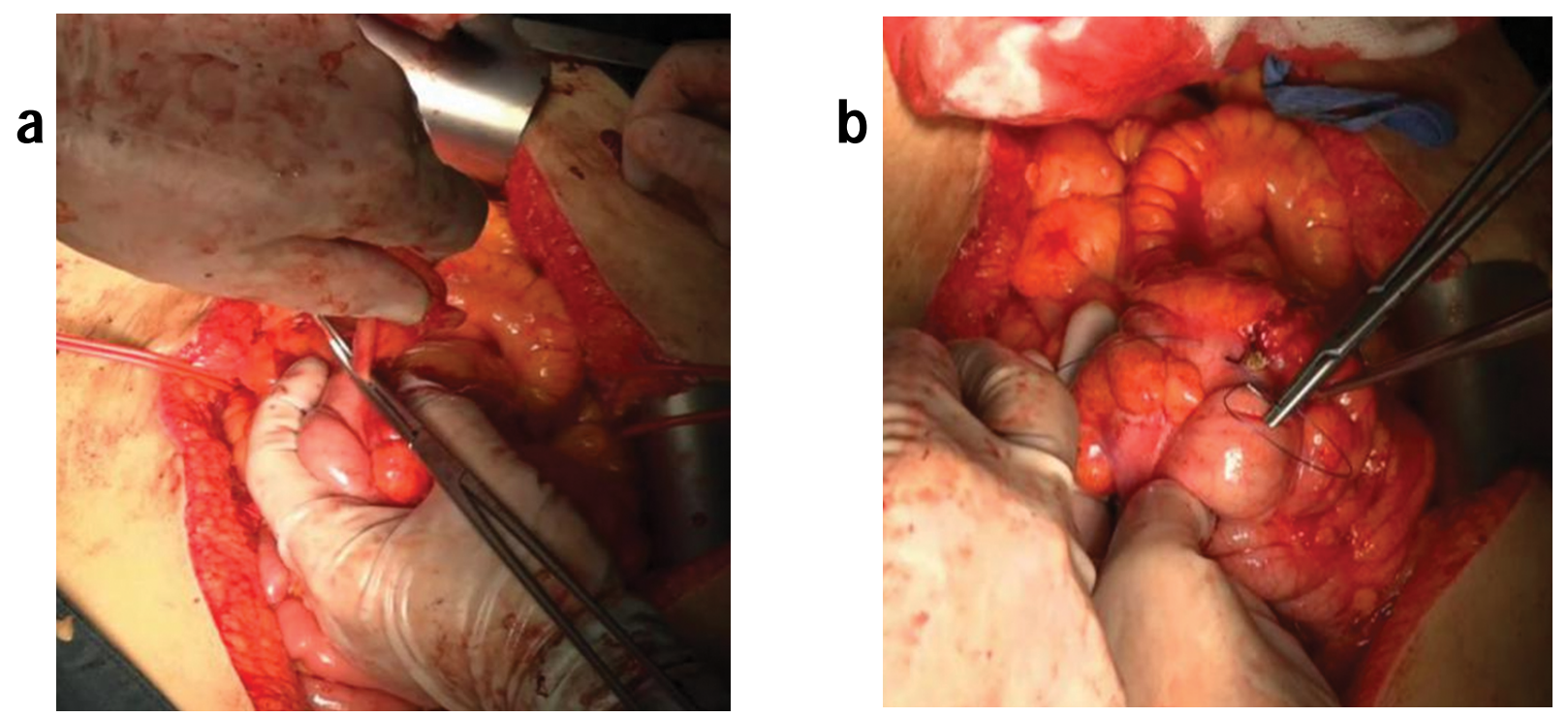

Figure 19. Appendectomy (a) before (b) after (routine operation)

(ileocecum and ascending colon are not resected in this case)

When it is mandatory the splenectomy is performed depending on the status of tumor invasion ${ }^{22}$ (Figure 20), greater attention is paid while ligating and dividing the splenic vessels to avoid traumatizing the tail and body of the pancreas. Often, when there is a significant disease in the hilum of the spleen, the posterior approach is used where at first the spleen is completely mobilized and then splenic vessels are approached and dissected posterolaterally, it is safer and can help in avoiding injury to the pancreas. Splenectomy is not performed in cases where the spleen is not involved by tumor ${ }^{22}$.

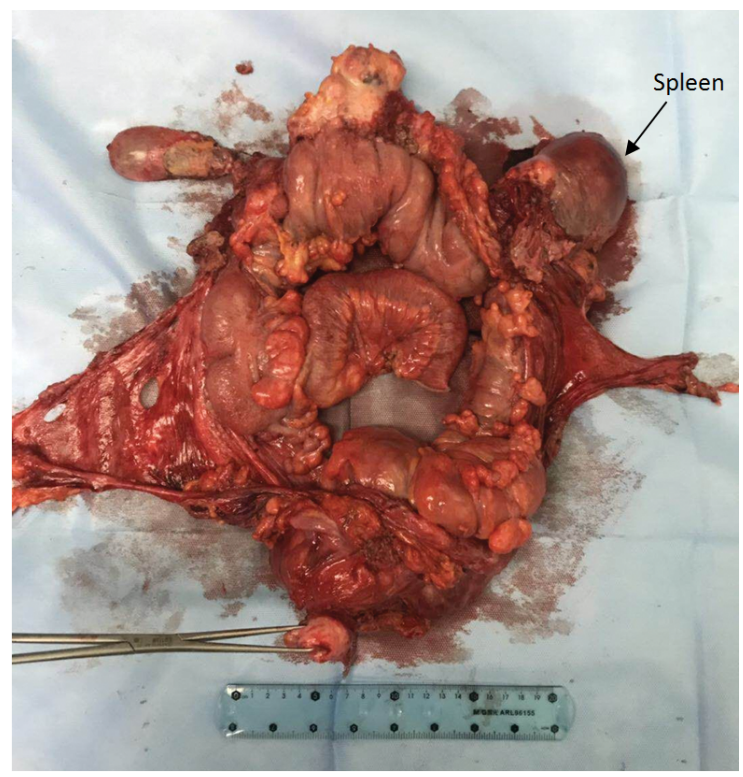

Figure 20. Splenectomy along en bloc with uterus, adnexa, partial rectum, total colon, omentum, pelvic peritoneum, prevesical peritoneum, abdominal wall peritoneum, bilateral paracolic gutter peritoneum, right and left subphrenic peritoneum (another case). 
Pelvic and para-aortic lymph node sampling is an integral part of the staging system of ovarian cancer as well the systematic lymphadenectomy in patients affected by early and advanced-stage ovarian cancer ${ }^{23}$. It is meaningful to have a radical tumor resection in the abdominal cavity, if the patient is in good physical condition, age $\leq 75$ years old, and the tumor is invasive epithelial carcinoma. Lymphadenectomy is associated with greater 5-year overall survival in early and advanced stages of ovarian cancer, also have shown the effect on progression-free survival and recurrence rate in patients with advanced stage ovarian cancer ${ }^{24}$.

For bilateral para-aortic and pelvic lymphadenectomy, abdominal aorta is exposed to the level of renal vessels, lymph node dissection is performed by stripping the nodal tissue para-caval and para-aortic bilaterally to the level of the renal vessels (Figure $21 \mathrm{a}, \mathrm{b}, \mathrm{c}, \mathrm{d}$ ). The pelvic lymph nodes dissection is performed bilaterally by removing the lymph nodes overlying and anterolateral to the common iliac vessel, overlying and medial to the external iliac vessel, overlying and medial to the hypogastric vessels, and from the obturator fossa at a minimum anterior to the obturator nerve, and the ligation of large lymphatic vessels is performed.
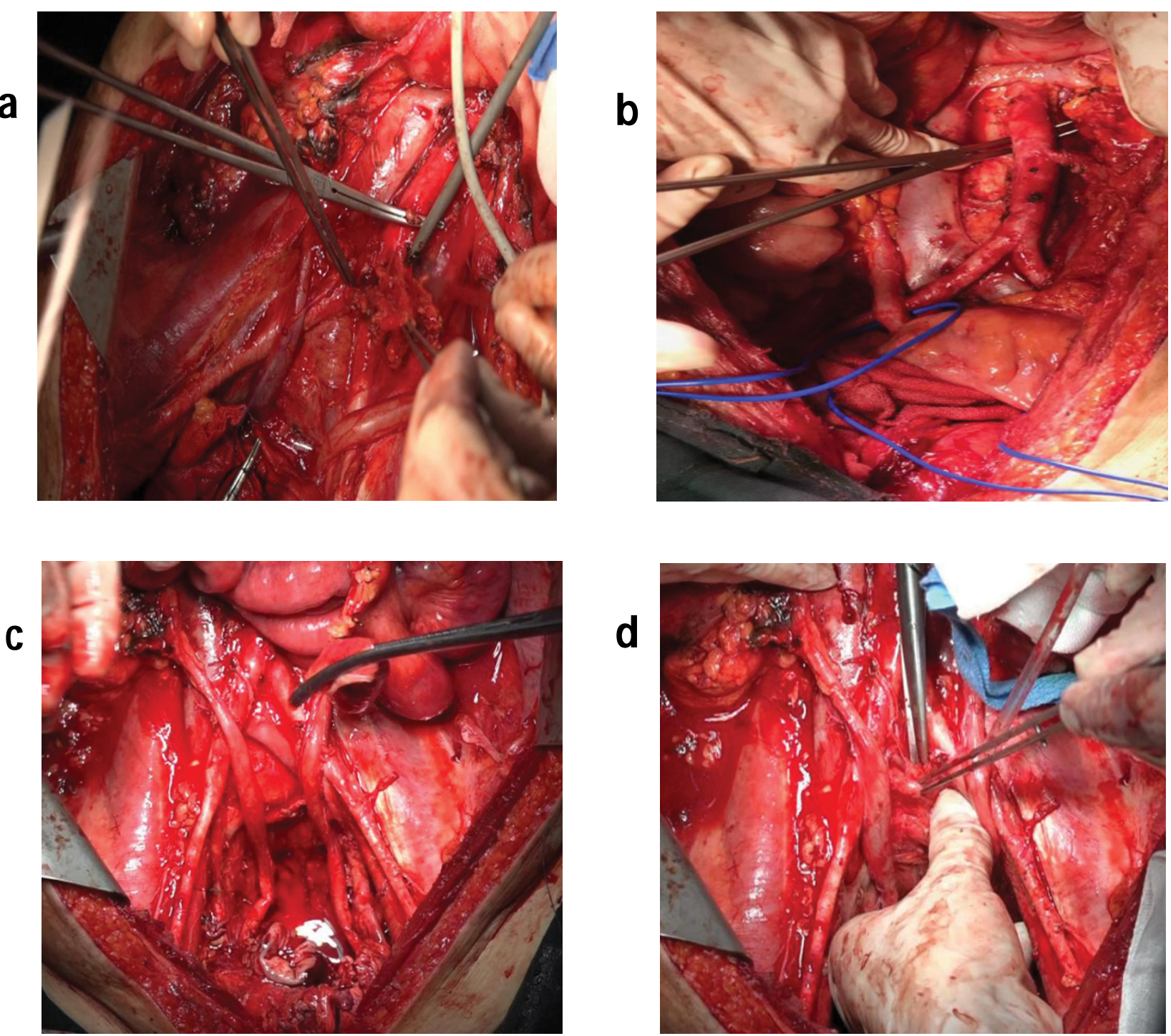

Figure 21. Para-aortic lymphadenectomy to the level of renal vessels (a) before (b) after, total resection of the pelvic lymph node (c) right side (d) left side. 
No macroscopic residual tissue is seen in the abdominal and pelvic examination at the end of primary CRS-R0, which makes it to be tumor-free state (Figure 22a). Abdominal drainage is placed but can be omitted (Figure 22b). Once the gauze and other equipment have been counted repeatedly, the incision is closed in layers (Figure 22c).
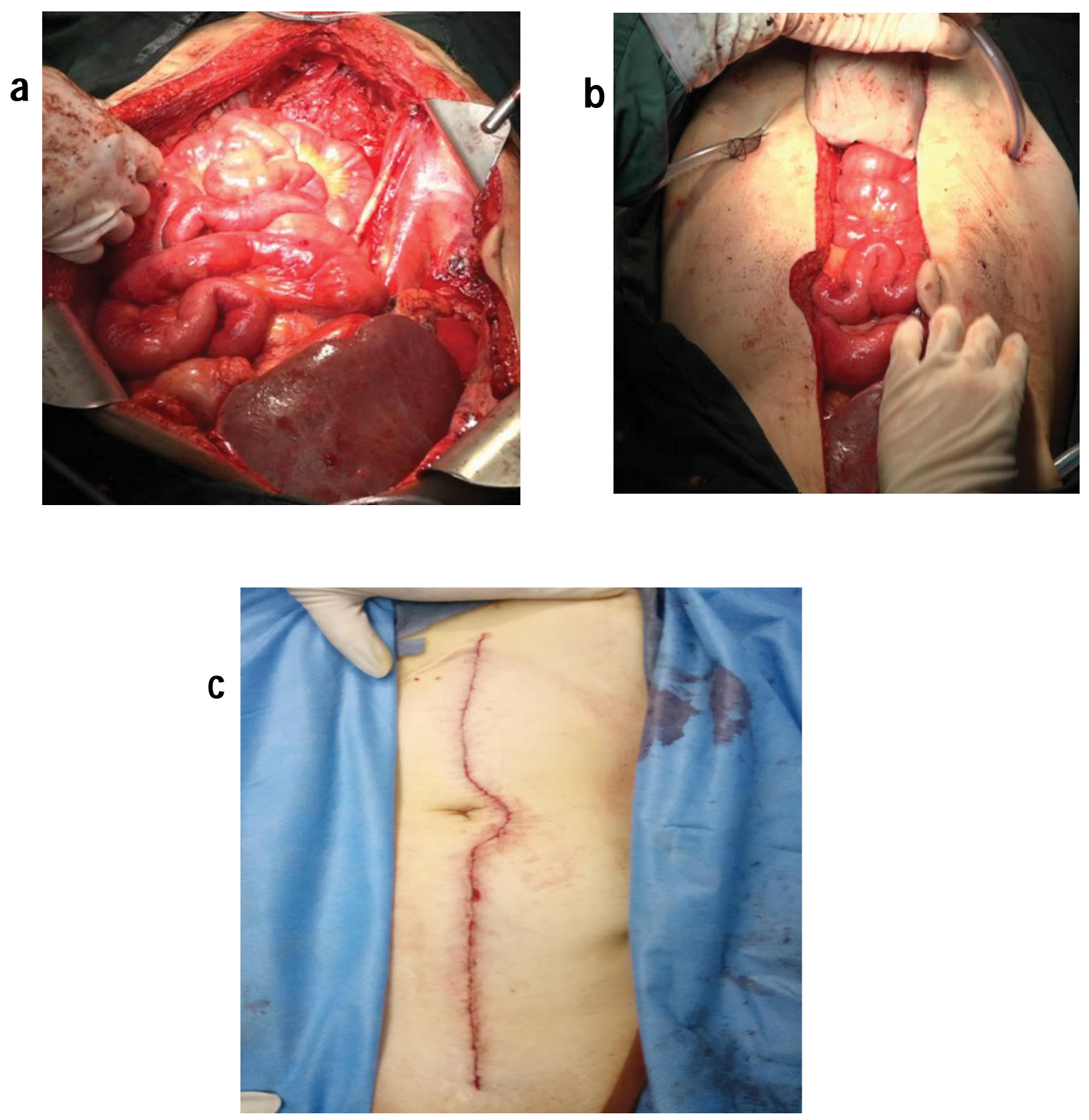

Figure 22. (a) ovarian cancer CRS with R0 (b) place abdominal drainage (c) incision closure.

Surgical specimen is displayed at the end of surgery, where all resected tissues can be seen (Figure 23). 

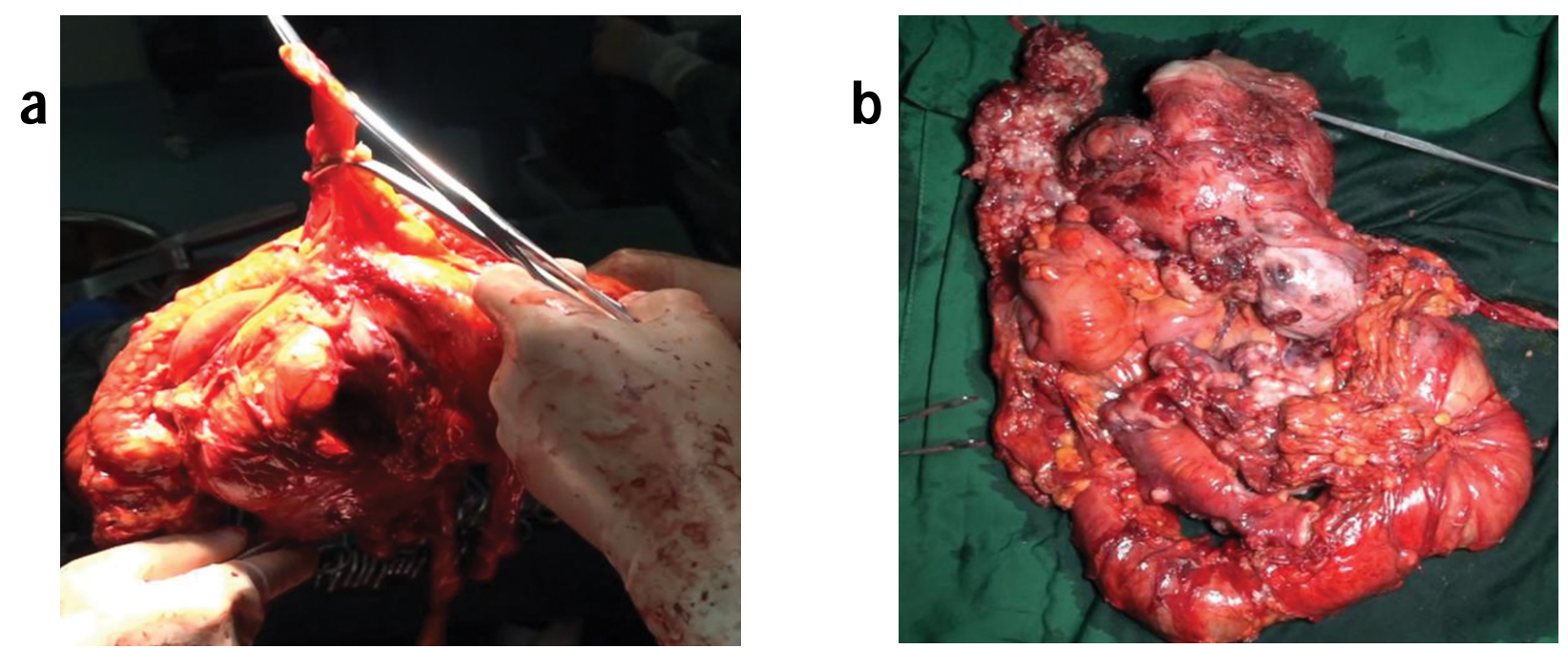

Figure 23. Surgical specimens: uterus, bilateral adnexa, partial rectum, total colon, partial ileum, omentum, pelvic wall peritoneum, prevesical peritoneum, abdominal wall peritoneum, bilateral paracolic gutter peritoneum, right diaphragmatic peritoneum.

\section{Conclusion}

Surgical staging and resection of the tumor and other invaded tissues have a major impact on the prognosis and survival in the management of ovarian cancer respectively. The central objective of the surgery is maximal/ complete cytoreduction leaving no residual/macroscopic disease behind instead of saying that $1-2 \mathrm{~cm}$ residual disease is still left in situ which is not the complete cytoreduction, and the former has proven to give a superior overall survival rate and combining with adjuvant IP or IV chemotherapy has shown to have prolonged progressionfree survival as well. The management of malignant epithelial tumors requires the support of a multidisciplinary team with careful case selection. The surgeon expertise and being comfortable in operating on all areas of the abdominal cavity and familiarity with the anatomy of various regions is also essential for obtaining optimal results, making CRS potentially curative with controlled postoperative mortality-morbidity and adequate impact of longterm quality of life. Since ultraradical procedures are routinely required, centers embracing an aggressive surgical paradigm have the highest success rates. Consistent referral of patients with apparent advanced ovarian cancer to gynecologic oncologists at expert centers may be the best means currently available for improving overall survival. When not feasible, cytoreduction to achieve as minimal residual disease as possible should be attempted using surgical judgment to balance potential perioperative risks and benefits, as each incremental decrease in residual disease below $1 \mathrm{~cm}$ may be associated with an improvement in overall survival. We are looking forward for better strategies and techniques to reduce the cancer burden and agony patients have to go through in advanced stages of disease.

\section{References}

1. Siegel RL, Miller KD, Jemal A. Cancer statistics. CA Cancer J. Clin. 2019; 69: 7-34.

2. Sugarbaker PH. Management of peritoneal metastases - Basic concepts. J BUON 2015;, 20(Suppl 1): S2-11.

3. Lambert LA. Looking up: Recent advances in understanding and treating peritoneal carcinomatosis. CA Cancer J Clin 2015; 65(4): 284-98.

4. Bao P, Bartlett D. Surgical techniques in visceral resection and peritonectomy procedures. Cancer J 2009; 15(3): 204-11.

5. Javellana M, Hoppenot C, Lengyel E. The road to long-term survival: Surgical approach and longitudinal treatments of long-term survivors, Gynecologic Oncology, 2018, j. ygyno. 2018.11.007. 
6. Zappa L, Sugarbaker PH. Compartment syndrome of the leg associated with lithotomy position for cytoreductive surgery, 2007, J Surg Oncol 96: 619-23.

7. Rosemarie Kearsley, Sine' ad Egan, Conan McCaul, 2018, Anaesthesia for Cytoreductive Surgery with Hyperthermic Intraperitoneal Chemotherapy (HIPEC),

Available: https://www.wfsahq.org, accessed 02 February 2019.

8. Shiralkar SP, Kerr P, Scott J, et al. Anaesthetic management of patients undergoing cytoreductive surgery with hyperthermic intraperitoneal chemotherapy for pseudomyxoma peritonei: a retrospective audit. Anaesth Intensive Care 2017; 45(4): 490-8.

9. Chang SJ, Bristow RE. Surgical technique of en bloc pelvic resection for advanced ovarian cancer. J Gynecol Oncol 2015; 26(2):155.

10. Martina Aida Angeles, Carlos Martinez-Gómez, Alejandra Martinez, et al, En bloc pelvic resection for ovarian carcinomatosis: Hudson procedure in 10 steps, 2018, j.ygyno. 2018.12.006.

11. Mourton SM, Temple LK, Abu-Rustum NR, et al. Morbidity of rectosigmoid resection and primary anastomosis in patients undergoing primary cytoreductive surgery for advanced epithelial ovarian cancer. Gynecol Oncol 2005; 99(3): 608-14.

12. Yildirim Y, Ertas IE, Nayki U, et al. Enbloc pelvic resection with concomitant rectosigmoid colectomy and immediate anastomosis as part of primary cytoreductive surgery for patients with advanced ovarian cancer. Eur J Gynaecol Oncol 2014; 35(4): 400-7.

13. Sonnendecker EW, Beale PG. Rectosigmoid resection without colostomy during primary cytoreductive surgery for ovarian carcinoma. Int Surg 1989; 74(1):10-12.

14. Sugarbaker PH. Avoiding Diverting Ileostomy in Patients Requiring Complete Pelvic Peritonectomy. Ann Surg Oncol. 2015.

15. Dagbert F, Passot G, Glehen O, Bakrin N. Glisson capsulectomy for extensive superficial liver involvement in peritoneal carcinomatosis (with video). J Visc Surg 2015; 152(5): 332-3.

16. Bacalbasa N, Dima S, Brasoveanu VDavid L, et al. Liver resection for ovarian cancer liver metastases as part of cytoreductive surgery is safe and may bring survival benefit. World J Surg Oncol 2015; 13: 235.

17. Gasparri ML, Grandi G, Bolla D, et al. Hepatic resection during cytoreductive surgery for primary or recurrent ovarian cancer. J Cancer Res Clin Oncol 2015; 1-12.

18. Pathiraja PNJ, Garruto-Campanile R, Tozzi R. Diaphragmatic peritonectomy versus full thickness diaphragmatic resection and pleurectomy during cytoreduction in patients with ovarian cancer, Int J Surg Oncol 2013; 876150 .

19. Son JH, Kong TW, Paek J, et al. Perioperative outcomes of extensive bowel resection during cytoreductive surgery in patients with advanced ovarian cancer. J Surg Oncol 2019. doi: 10.1002/jso.25403.

20. Cordeiro Vidal G, Croce S, Guyon F, et al. Total Infragastric Omentectomy Including the Vascular Perigastric Arcade in Patients With Advanced Serous Ovarian Tumors. Int J Gynecol Cancer 2017; 27(2): 252-7.

21. Wong LF, Wahab NA, Gleeson N, Appendectomy with cytoreductive surgery for ovarian and type 2 endometrial carcinoma. Eur J Gynaecol Oncol. 2014; 35(2): 143-8.

22. Sun H, Bi X, Cao D, et al. Splenectomy during cytoreductive surgery in epithelial ovarian cancer. Cancer Manag Res 2018; 10: 3473-82.

23. Angioli R, Plotti F, Palaia I, et al. Update on lymphadenectomy in early and advanced ovarian cancer. Curr Opin Obstet Gynecol. 2008; 20(1): 34-9.

24. Zhou J, Shan G, Chen Y, et al. The effect of lymphadenectomy on survival and recurrence in patients with ovarian cancer: a systematic review and meta-analysis. Jpn J Clin Oncol. 2016; 46(8): 718-26. 